\title{
A critical appraisal on whether a traumatic pneumothorax should be managed conservatively
}

\author{
Authors: Hardeep Sahota, Joshua Hinton, Emma Worley, Ashleigh Milner, Ellen Kay, Sarah Badger
}

\section{Aims}

$>$ Discuss when it is appropriate to use conservative management for a traumatic pneumothorax.

> Highlight the complications associated with conservative management.

$>$ Discuss when it is appropriate to intervene with a tube thoracostomy.

$>$ Highlight the complications associated with the use of a tube thoracostomy.

\section{Methods}

A literature search was conducted to identify the published research; a critical appraisal of the research identified was then conducted using CASP tools.

\section{Results}

\section{Reasons to use a drain}

$>$ If the pneumothorax is initially $>1.5 \mathrm{~cm}$ in size, the patient needs a chest drain insertion.

$>$ If the patient is haemodynamically unstable or has a multitude of other injuries, the patient needs a chest drain.

> If the patient requires IPPV (intermittent positive pressure ventilation), a chest drain is always required, due to increased risk of a tension pneumothorax developing.

> If the patient is symptomatic from the traumatic pneumothorax, especially if they are older.

$>$ Only $10 \%$ of patients initially managed conservatively then go onto have a drain inserted.

\section{Reasons not to use a drain}

$>$ Small to moderate-sized pneumothorax.

> Some types of pneumothorax can resolve spontaneously; Patients are very unlikely to deteriorate clinically if monitored when undergoing conservative management.

> CT scan usage increasing; CT has become cheaper and therefore more widely utilised CT is available 24/7.

> Shorter average hospital stay - 12.9 vs 17.6 days for conservative vs drain.

\section{Conclusions}

> Conservative management can be the appropriate treatment in small to moderate-sized pneumothorax, with $1.5 \mathrm{~cm}$ being the cut-off point.

> Size cannot be the only factor to determine the best treatment; is the patient haemodynamically stable with few symptoms?

> Other factors which must be included are if there are other injuries present, or whether they may need IPPV, as these can increase the risk of complications such as a tension pneumothorax.

> Clear guidelines need to be established so that junior medical staff can refer to a policy which can aid their clinical decision-making process. 